\title{
Conhecimento dos pescadores artesanais sobre a composição da dieta dos pacus (Characiformes: Serrasalmidae) na Floresta Nacional do Amapá,rio Araguari, Amapá, Brasil
}

\author{
Aldilene Lobato dos Santos ${ }^{1}$ \\ Fabiana Calacina da Cunha ${ }^{2 *}$ \\ Maria Gercilia Mota Soares ${ }^{3}$ \\ Luiza Prestes de Souza ${ }^{1}$ \\ Alexandro Cezar Florentino ${ }^{4}$ \\ ${ }^{1}$ Universidade do Estado do Amapá, Macapá - AP, Brasil \\ ${ }^{2}$ Universidade Federal do Amazonas \\ Programa de Pós-graduação em Ciências Pesqueiras nos Trópicos \\ Avenida General Rodrigo Otávio, 6200, Coroado I, Cep 69077-000, Manaus - AM , Brasil \\ ${ }^{3}$ Instituto Nacional de Pesquisas da Amazônia, Manaus - AM, Brasil \\ ${ }^{4}$ Universidade Federal do Amapá, Macapá - AP, Brasil \\ * Autor para correspondência \\ cunha.fabianac@gmail.com
}

Submetido em 08/04/2015

Aceito para publicação em 16/02/2016

\section{Resumo}

O conhecimento ecológico tradicional (CET) dos pescadores é uma ferramenta importante para obtenção de informações ecológicas sobre o ambiente que vem sendo cada vez mais utilizada na conservação dos recursos naturais. Nesse contexto, o referido trabalho descreve o CET do pescador sobre a composição da dieta e local de pesca dos pacus (Characiformes: Serrasalmidae) Myloplus sp., Myloplus asterias, Myloplus ternetzi, Tometes trilobatus, Prosomyleus rhomboidalis e Mylesinus paraschomburgkii na Floresta Nacional do Amapá (FLONA-AP). Para a coleta das informações, foram realizadas entrevistas semiestruturadas ( $\mathrm{n}=20$ pescadores) e turnê-guiada. Segundo os pescadores, os pacus se alimentam de frutos/sementes, fragmentos vegetais, insetos e perifíton. No inverno, consomem frutos/sementes e fragmento de vegetais. No verão, além de frutos/sementes e fragmentos de vegetais, foi observada presença de perifíton e insetos. Comparando-se as informações dos pescadores com a literatura científica, observam-se similaridades para o período de inverno. Mas, no verão, os pescadores relatam a ingestão de perifíton e invertebrados não mencionados na literatura. Os locais de pesca são caracterizados como sazonais: no inverno a pesca ocorre nos varjões e, no verão, nas pedreiras e calha principal do rio. Os pescadores possuem um conhecimento detalhado sobre a alimentação dos pacus e o grau de refinamento do conhecimento é fundamental para a conservação na FLONA-AP.

Palavras-chave: Áreas protegidas; Conhecimento ecológico tradicional; Dieta de pacus; Locais de pesca; Pesca artesanal 


\title{
Abstract
}

\begin{abstract}
Knowledge of artisanal fishermen about the diet composition of pacu (Characiformes: Serrasalmidae) in Amapá National Forest, Araguari River, Amapá, Brazil. The traditional ecological knowledge (TEK) of fishermen is an important tool for obtaining ecological information about the environment and is being increasingly used in the conservation of natural resources. This study describes the TEK of fishermen about the diet composition and fishing places of pacu (Characiformes: Serrasalmidae), Myloplus sp., Myloplus asterias, Myloplus ternetzi, Tometes trilobatus, Prosomyleus rhomboidalis and Mylesinus paraschomburgkii, in the National Forest of Amapá (FLONA-AP). To collect the information, interviews were conducted with fishermen $(n=20)$ and a guided tour was given. According to the fishermen, pacu feed on fruits/seeds, plant debris, insects and periphyton. In the winter the fish consume fruits/seeds and plant fragments, and in the summer they eat fruits/ seeds, plant fragments, periphyton and insects. When comparing the information provided by the fishermen to scientific literature there are similarities for the winter period; however, for summer the fishermen reported the consumption of periphyton and invertebrates, which is not mentioned in the literature. The fishing places were seasonal. In the winter, fishing occurs in varjões (seasonally flooded areas) and in the summer in quarries and in the main river channel. The fishermen have detailed knowledge about the feeding of pacu and this knowledge is essential for conservation in FLONA-AP.
\end{abstract}

Key words: Artisanal fishing; Pacu diet; Places to fish; Protected areas; Traditional ecological knowledge

\section{Introdução}

O conhecimento ecológico tradicional (CET) pode ser entendido como o conhecimento, práticas e crenças que as populações humanas possuem sobre o ambiente natural. Tais conhecimentos foram construídos e incorporados por um grupo de pessoas, por meio de práticas adaptativas, a partir do contato direto com os recursos naturais ou por gerações, via transmissão cultural (RUDDLE, 1993; BERKES, 1999). Por esse motivo, o CET deve ser analisado a partir das relações das atividades práticas das populações, ou seja, do seu contato direto com o sistema natural e com o sistema de crenças do grupo cultural no qual se inserem (BERKES et al., 2000). Nas últimas décadas, o CET tem ampliado informações sobre animais e plantas. Isso é importante para a proteção de hábitats, espécies e para a manutenção da biodiversidade por meio da sua inclusão em ações de conservação (JOHANNES, 1993; FRASER et al., 2006; SILVANO et al., 2008).

No caso dos pescadores, a existência de uma relação estreita com a dinâmica das águas e a ictiofauna aumenta a interdependência das estratégias de pesca aos ciclos naturais, possibilitando a aplicação dos conhecimentos ecológicos no manejo e uso do recurso pesqueiro, necessários à subsistência e/ou geração de renda (COSTA-NETO; MARQUES, 2000; DIEGUES,
2000; BEGOSSI, 2004; RAMIRES et al., 2007). Além disso, é reconhecida a importância do conhecimento tradicional, pois, em alguns casos, pode ser base exclusiva de informações importantes relativas às condições passadas ou sobre áreas que sofreram alterações ambientais (JOHANNES, 1998).

Além disso, o saber dos pescadores sobre os peixes revela sua utilidade quando tem a possibilidade de se tornar indicadores da biodiversidade e da ecologia da ictiofauna local (BEGOSSI, 1999; 2004), principalmente porque possibilitam o acesso rápido às informações importantes para a conservação do recurso pesqueiro e também por somarem-se às informações técnico-científicas para o norteamento do processo de gestão dos recursos naturais, inclusive em Unidades de Conservação - UC (BARAÚNA, 2000; PEDROSO JUNIOR; SATO, 2005; BARROS, 2012; ROSSONI et al., 2014).

$\mathrm{Na}$ região amazônica, o saber ecológico dos pescadores tem sido uma abordagem importante nos estudos sobre a bioecologia de peixes. No Amazonas e Pará, têm destaque estudos sobre a alimentação (SILVA; MONTAG, 2003; BATISTELLA et al., 2005; REBELO et al., 2010; BARROS, 2012; LIMA; BATISTA, 2012), reprodução (LIMA; BATISTA, 2010; CUNHA et al., 2010), migração (BARROS; RIBEIRO, 2005) e distribuição dos peixes nos hábitats (BARBOZA; 
PEZZUTI, 2011; CARVALHO-JÚNIOR et al., 2011; BARROS, 2012).

Apesar da importância do CET que os pescadores possuem, ainda é incipiente o seu uso no Amapá. Algumas informações podem ser obtidas em monografias e dissertações (DAADDY, 2012; SANTOS, 2012). As informações baseadas no saber de pescadores podem ser potencialmente importantes para nortear o processo de gestão de uma das Unidades de Conservação mais antigas do estado, a Floresta Nacional do Amapá (FLONA-AP).

A Floresta Nacional do Amapá é a primeira UC de uso sustentável do estado do Amapá, possuindo uma área de 412 mil hectares de floresta tropical, considerada a quinta maior do estado. A FLONA-AP está inserida no Corredor da Biodiversidade do Amapá, o qual faz parte do Escudo das Guianas, o maior conjunto de áreas protegidas de florestas tropicais do mundo (BRANDÃO; SILVA, 2008; ICMBIO, 2011). Os recursos hídricos da FLONA-AP (vários igarapés e o rio Araguari) são de grande importância para a pesca, a qual é exercida pela maioria dos moradores da própria $\mathrm{UC}$ e pelos pescadores registrados na Colônia de Pescadores Z-16, em Porto Grande (SOARES et al., 2012). Assim, o presente trabalho propôs-se a descrever o Conhecimento Ecológico Tradicional (CET) dos pescadores sobre a dieta e local de pesca de seis etnoespécies ${ }^{1}$ de pacus (Characiformes: Serrasalmidae), alvos da pesca comercial no rio Araguari, dentro da FLONA-AP.

\section{Materiais e Métodos}

\section{Área de estudo}

A Floresta Nacional do Amapá (FLONA-AP), criada no ano de 1989, está localizada na região central do estado e tem uma área aproximada de 412 mil hectares. Seus limites geográficos são definidos pelos rios Falsino, Araguari e Mutum e está inserida nas áreas municipais de Ferreira Gomes, Pracuúba e Amapá (ICMBIO, 2011) (Figura 1). A vegetação é diversa e já foram registradas 143 espécies vegetais (PEREIRA et al., 2007), sendo caracterizada por elementos de floresta tropical úmida, de cerrado e de várzea (BEZERRA et al., 1990; IBGE, 2010). De acordo com censo realizado em 2011, 24 pessoas residem no interior da UC, desenvolvendo atividades de agricultura, extrativismo vegetal e pesca (ICMBIO, 2011).

FIGURA 1: Localização geográfica da Floresta Nacional do Amapá e município de Porto Grande, Amapá.

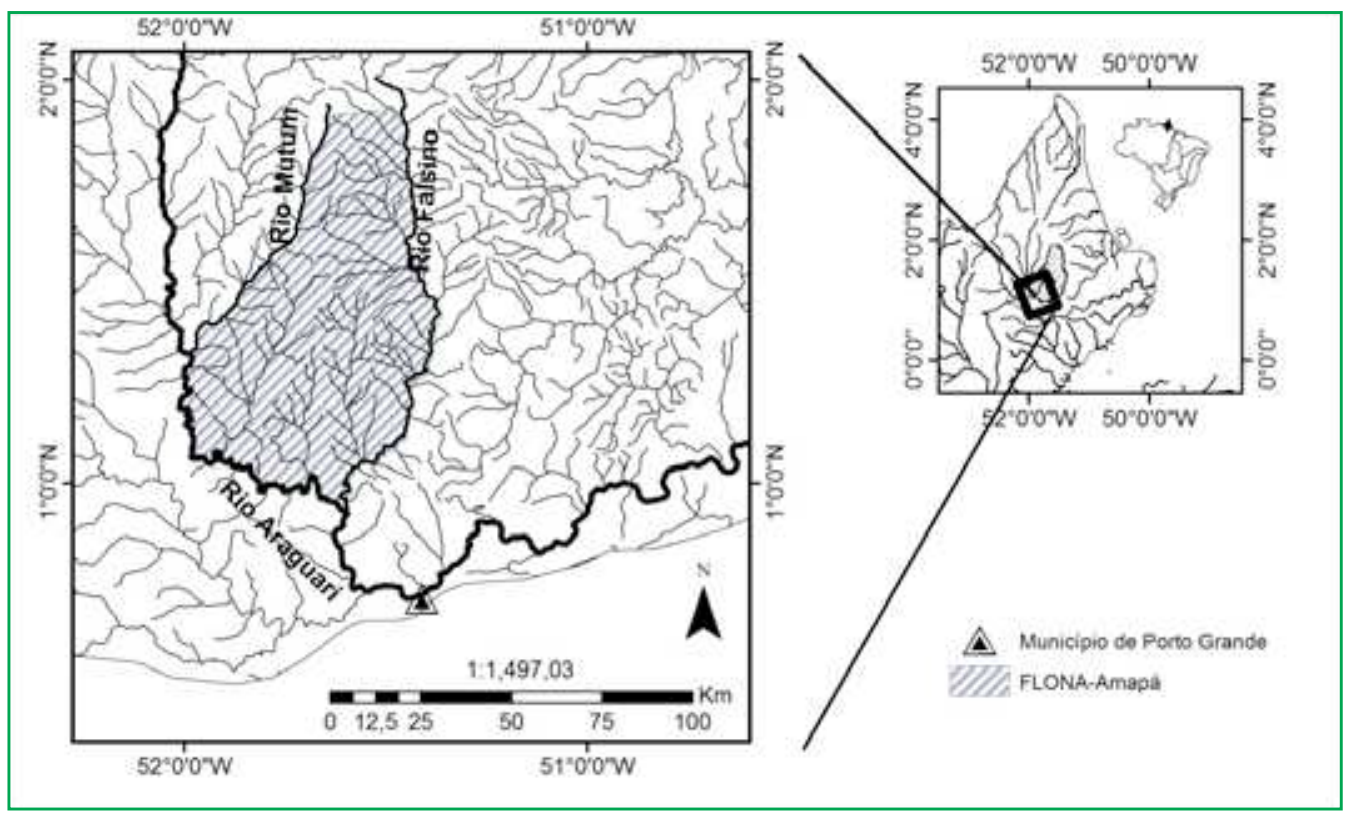

1 Etnoespécie refere-se à denominação popular dos tipos de pacu citados pelos pescadores entrevistados. 
No município de Porto Grande, reside a maior parte dos pescadores que atuam na área do rio Araguari, dentro da FLONA-AP, estando registrados na Colônia de Pescadores Z-16. Este município, localizado ao sul do estado (Mesorregião Sul), a 108 km de Macapá, possui população composta por 16.825 habitantes, dos quais $63,95 \%$ habitam na zona urbana e $36,05 \%$ na zona rural (IBGE, 2010).

No rio Araguari é possível identificar duas estações climáticas bem definidas, denominadas pelos pescadores de "inverno" (período chuvoso) e "verão" (período de estiagem) (Figura 2). O "inverno" é marcado pela alta pluviosidade entre fevereiro e maio $(862-323 \mathrm{~mm}$ de chuvas), quando ocorre elevação das cotas do rio $(478-520 \mathrm{~cm})$ que alaga as áreas marginais, favorecendo a dispersão dos peixes. No "verão", a retração das águas (314-359 cm de cota) e a baixa pluviosidade (116-29 mm de chuvas) reduzem o volume de água, obrigando os peixes a permanecerem no canal principal do rio, especialmente nos locais mais profundos (Figura 2).

FIGURA 2: Variação da cota $(\mathrm{cm})$ e precipitação mensal $(\mathrm{mm})$ em 2012 do rio Araguari na estação de Porto Platon.

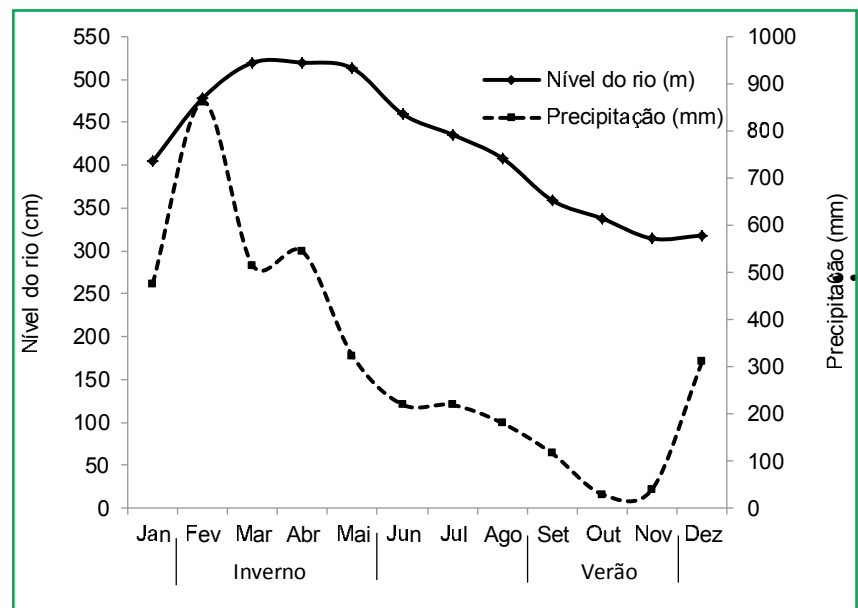

Fonte: Agência Nacional de Águas (ANA) < http://www.ana.gov.br >.

\section{Coleta de dados}

Para a realização deste estudo, foram selecionadas seis etnoespécies de pacus, que são as mais consumidas e comercializadas nas feiras de Porto Grande (SOARES et al., 2012): "pacu-branco" (Myloplus sp.), "pacumafurá" (Myloplus asterias), "pacu-ferro" (Myloplus ternetzi), "pacu-curupeté" (Tometes trilobatus), "pacu-cumaru" (Prosomyleus rhomboidalis) e "pacuflaviano" (Mylesinus paraschomburgkii). A identificação científica e popular dessas espécies foi realizada no âmbito do projeto Bioecologia e etnoecologia da ictiofauna da Floresta Nacional do Amapá (SOARES et al., 2012), o qual utilizou métodos da pesquisa etnoecológica, pescarias experimentais, dados de desembarque pesqueiro e consulta a especialistas para a identificação taxonômica dos peixes. No caso dos pacus, a identificação foi realizada por Michel Jegu - Institut de Recherche pour le Développement (IRD-France) e MSc. Marcelo Costa Andrade (Doutorando/UFPA), especialistas nesse grupo taxonômico.

A coleta de dados foi realizada no período de março a maio de 2012, por meio de entrevistas $(n=20)$ com pescadores da FLONA-AP e da Colônia de Pescadores Z-16 de Porto Grande, utilizando um roteiro semiestruturado de questões. Os pescadores foram selecionados com base em dois critérios: realizar a atividade pesqueira para consumo e/ou comercialização e reconhecida experiência na pesca confirmada pelos demais entrevistados. Esses critérios foram definidos para a identificação dos especialistas nativos (MARQUES, 1995). O método reconhecido como bola de neve "snowball" (BAILEY, 1987) foi utilizado para a indicação dos informantes, no qual o pescador entrevistado sugere ao final da entrevista outro pescador. Quando os nomes indicados se repetiram, encerrou-se a realização das entrevistas. O roteiro de questões continha perguntas sobre o perfil dos entrevistados, idade, gênero, escolaridade e modalidade de pesca. Em seguida, questões sobre a alimentação de cada etnoespécie no "inverno" e "verão" e, por fim, qual o local de pesca de cada etnoespécie nos dois períodos.

Após a tabulação dos dados e análise das informações, utilizando a estatística descritiva, foram listados os principais itens alimentares mencionados pelos pescadores. Posteriormente, foi aplicada a técnica turnê-guiada (ALBUQUERQUE; LUCENA, 2004), na qual três informantes e pesquisadores foram a campo para coleta de amostras botânicas citadas durante a entrevista, registrando informações sobre o local de ocorrência, período de frutificação e floração. $\mathrm{Na}$ coleta, foi realizado o registro fotográfico das espécies 
e de partes dela, tais como frutos e folhas. As amostras coletadas foram prensadas (prensas provisórias) e, em alguns casos, acondicionadas em sacos e recipientes plásticos, no caso dos frutos (ROTTA et al., 2008). O acervo de fotografias e as amostras foram levados para os especialistas Luciano Araújo Pereira, Driss Wagner Pantoja Pena e Breno Marques da Silva e Silva, que realizaram a identificação. Para esse trabalho não foram coletados exemplares de insetos, apenas realizada a observação direta durante a turnê-guiada, o que não permitiu a identificação em nível de espécie desse grupo.

\section{Resultados}

\section{Perfil dos pescadores entrevistados}

Os entrevistados $(\mathbf{n}=20)$, em sua maioria, são do sexo masculino ( $90 \%$ ), tendo uma idade média de 52,5 ( \pm 9 anos), variando entre 32 e 65 anos. Com relação à escolaridade, $45 \%$ possuem o ensino fundamental incompleto, $15 \%$ o ensino fundamental completo e apenas $5 \%$ dos entrevistados concluíram o ensino médio. Com relação à modalidade de pesca, a maioria pesca para comercializar (85\%). Portanto, a pesca é uma atividade econômica que vem sendo realizada na FLONA-AP.

\section{Composição da dieta}

Segundo os pescadores, os seis tipos de pacu têm a dieta constituída por quatro principais tipos de alimentos. Entre os frutos/sementes, foram mencionados o jenipapo (Genipa americana) (29\%), a andiroba (Carapa guianensis) (23\%), o sarão (Alchornea cf. discolo) (16,9\%), a ginja (Prumus lusitanica) $(16,1 \%)$, a seringa (Hevea brasilienses) (5,6\%), o camuti (Mouriri cf. acutiflora) (2,8\%), o melão (Vilbrandia cf. verticillata) $(2,8 \%)$, o taperebá (Spondias mombin) $(1,3 \%)$, o ingá (Ingá capitada) (1\%) e a imbaúba (Cecropia pachystachya) (1\%). Segundo os pescadores, o jenipapo e a andiroba estão presentes na dieta dos pacus no "inverno" e "verão", exceto para o "pacu-flaviano". Os fragmentos vegetais, constituídos, principalmente, por frutos não identificados, incluíram fragmentos de folhas, flores e raízes. Na categoria insetos, destacouse a presença dos cupins (Isoptera) e gafanhotos
(Orthoptera). O "limo" é a denominação utilizada pelos pescadores locais para o perifíton e algas.

A principal diferença na composição da dieta dos pacus está relacionada à sazonalidade de oferta dos recursos de acordo com os períodos. Segundo os pescadores, no "inverno" é alto o consumo de frutos/ sementes e fragmentos vegetais, enquanto que no "verão", além de frutos/sementes e fragmentos de vegetais, os pescadores relatam o consumo de perifíton e insetos (Figura 3)

FIGURA 3: Conhecimento dos entrevistados sobre os principais itens alimentares das etnoespécies pacus no inverno (Inv) e verão (Ver) no rio Araguari, FLONA-AP (Frequência das citações dos entrevistados, $\mathrm{n}=20$ ).

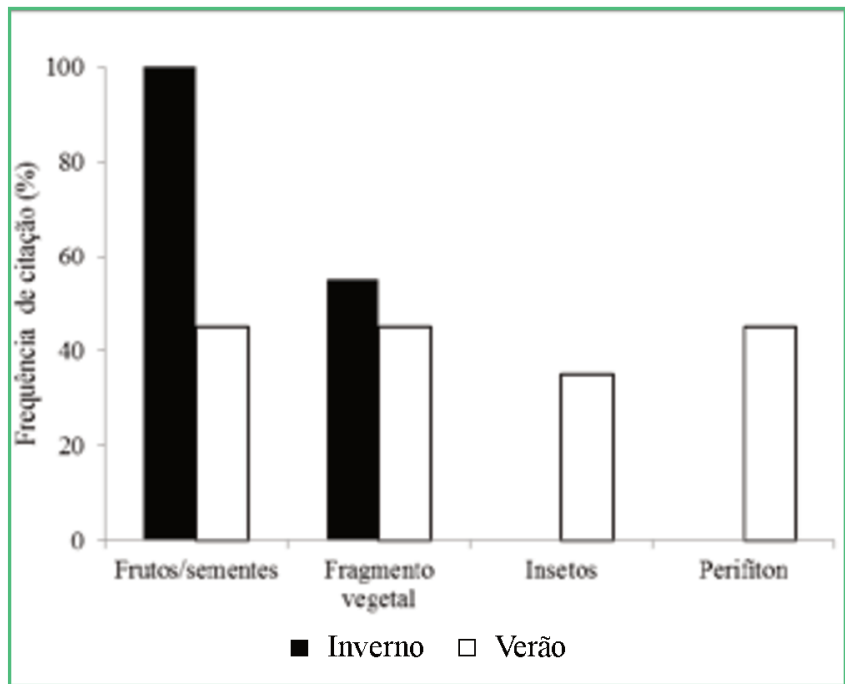

Em nível de etnoespécies, a composição da dieta no "inverno" relatada pelos pescadores é similar entre o "pacu-cumaru", "pacu-ferro", "pacu-mafurá", "pacubranco"e "pacu-curupeté", que ingerem, principalmente, os frutos/semente de jenipapo, ginga, sarão, seringa e andiroba. No entanto, o "pacu-flaviano" se diferencia dos demais nesse período pela ausência de citações de ingestão de jenipapo. No "verão", todos os pacus são relatados como ingerindo, também, insetos e perifíton.

Ao comparar o período de ingestão dos frutos pelos pacus citados nas entrevistas e o período de frutificação disponível na literatura científica, é possível verificar que a maior parte dos frutos tem frutificação sazonal, correspondendo aos períodos mencionados pelos pescadores. No entanto, para a andiroba, apesar de o registro na literatura descrever a frutificação entre março 
e maio (FREITAS et al., 2010; 2013), a ingestão deste, de acordo com o relato dos pescadores, ocorreu tanto no "inverno" quanto "no verão" (Tabela 1).

\section{Local de pesca das etnoespécies de pacus}

No rio Araguari, podem ser destacados seis locais de pesca que, atualmente, estão sendo utilizados pelos pescadores da Colônia de Pescadores Z-16 (Tabela 2). A descrição desses hábitats com base no conhecimento dos pescadores foi realizada preliminarmente por Soares et al. (2012):
1) Varjão: áreas de floresta nas margens do rio, inundadas no período do "inverno", com profundidade de aproximadamente $2 \mathrm{~m}$. É, segundo os pescadores, local de abrigo, alimentação e reprodução para peixes.

2) Pedreiras: são áreas com algumas pedras na calha principal do rio, sendo utilizadas frequentemente no período de "verão" para capturar pacus com o uso de caniço.

3) Calha do rio: áreas de água aberta do rio. No "inverno", a área fica maior, estendendo-se por causa da alagação, enquanto que no "verão", com a retração da água, essas áreas ficam mais restritas.

TABELA 1: Tabela de cognição comparada entre o CET dos pescadores sobre a ingestão de frutos e sementes pelas etnoespécies de pacu no período de inverno (Inv) e verão (Ver) no rio Araguari, FLONA-AP e o período de frutificação descrito na literatura científica.

\begin{tabular}{|c|c|c|c|c|c|c|c|c|}
\hline \multirow{2}{*}{ Itens } & \multicolumn{6}{|c|}{$\begin{array}{l}\text { Período de ingestão dos frutos/sementes de acordo } \\
\text { com CET dos pescadores }\end{array}$} & \multicolumn{2}{|c|}{$\begin{array}{l}\text { Período de frutificação de acordo com a } \\
\text { literatura científica }\end{array}$} \\
\hline & $\begin{array}{l}\text { Pacu- } \\
\text { cumaru }\end{array}$ & $\begin{array}{l}\text { Pacu- } \\
\text { ferro }\end{array}$ & $\begin{array}{c}\text { Pacu- } \\
\text { flaviano }\end{array}$ & $\begin{array}{l}\text { Pacu- } \\
\text { mafurá }\end{array}$ & $\begin{array}{c}\text { Pacu- } \\
\text { branco }\end{array}$ & $\begin{array}{c}\text { Pacu- } \\
\text { curupeté }\end{array}$ & Período & Autor \\
\hline Jenipapo & Inv, Ver & Inv & - & Inv, Ver & Inv, Ver & Inv, Ver & $\begin{array}{l}\text { Março a junho, pode } \\
\text { frutificar em outras épocas }\end{array}$ & Maia, 2001 \\
\hline Canuti & Inv & - & - & - & - & - & - & - \\
\hline Ginja & Inv & Inv & Inv & Inv & Inv & Inv & - & - \\
\hline Sarão & Inv & Inv & Inv & Inv & Inv & Inv & - & - \\
\hline Seringa & Inv & - & - & Inv & Inv & Inv & Agosto a maio & Maia, 2001 \\
\hline Andiroba & Ver & Inv, Ver & Inv & Inv, Ver & Inv, Ver & Inv, Ver & Março a maio & $\begin{array}{c}\text { Freitas et al.. 2010; } \\
2013\end{array}$ \\
\hline Taperebá & - & Inv & Inv & - & - & - & Novembro a março & Freitas et al.. 2010 \\
\hline Ingá & - & - & Inv & - & - & - & $\begin{array}{l}\text { Picos em março, maio, } \\
\text { agosto/setembro e outubro/ } \\
\text { janeiro }\end{array}$ & $\begin{array}{l}\text { Falcão e Clemente, } \\
\text { 2000; Silva et al., } 2007\end{array}$ \\
\hline Imbaúba & - & - & - & - & Inv & - & $\begin{array}{l}\text { Fevereiro a agosto/ } \\
\text { setembro a outubro }\end{array}$ & $\begin{array}{c}\text { Maia, 2001; Salman et } \\
\text { al., 2008; Silva Júnio et } \\
\text { al., } 2013\end{array}$ \\
\hline Melão & - & - & - & - & Inv & - & - & - \\
\hline
\end{tabular}

TABELA 2: Conhecimento dos entrevistados sobre os habitat das etnoespécies pacus no inverno (Inv) e verão (Ver) no rio Araguari, FLONA-AP. Frequência das citações dos entrevistados, n = 20).

\begin{tabular}{ccccccccccccc}
\hline \multirow{2}{*}{ Habitat } & \multicolumn{2}{c}{ Pacu-cumaru } & \multicolumn{2}{c}{ Pacu-ferro } & \multicolumn{2}{c}{ Pacu-flaviano } & \multicolumn{2}{c}{ Pacu-mafurá } & \multicolumn{2}{c}{ Pacu-branco } & \multicolumn{2}{c}{ Pacu-curupeté } \\
\cline { 2 - 14 } & Inv & Ver & Inv & Ver & Inv & Ver & Inv & Ver & Inv & Ver & Inv & Ver \\
\hline Varjão & 85 & - & 93,3 & - & 68,4 & - & 80 & - & 95 & - & 95 & - \\
Pedreiras & 5 & 50 & 6,7 & 53,3 & 10,5 & 77,8 & 6,7 & 61,5 & 5 & 50 & 5 & 62,5 \\
Calha do rio & 10 & 45 & - & 26,7 & - & 22,2 & 13,3 & 30,8 & - & 43,7 & - & 31,2 \\
Cachoeiras & - & 5 & - & 6,7 & - & - & - & 7,7 & - & 6,2 & - & 6,2 \\
Igarapé & - & - & - & 13,3 & - & - & - & - & - & - & - & - \\
Remanso & - & - & - & - & 21,0 & - & - & - & - & - & - & - \\
\hline
\end{tabular}


4) Cachoeiras: Áreas com pedras na calha principal do rio que apresentam significativa correnteza, sendo mais visíveis e mais acentuadas no "verão". Nas corredeiras, os pescadores relatam que ocorre aglomeração de peixes, como "pacu-curupeté" e "pacubranco". A pesca de caniço é comumente realizada em cima das pedras, na área da queda d'água da cachoeira.

5) Igarapé: corpos d'água de menor porte, que deságuam no rio, área de abrigo e alimentação de peixes.

6) Remanso: água parada próxima às áreas de corredeiras.

Com base nessa relação de hábitats, os pescadores relatam captura diferenciada das etnoespécies de pacus conforme as estações. No período de "inverno", o "pacucumaru”, "pacu-ferro", “pacu-flaviano”, "pacu-mafurá”, "pacu-branco" e "pacu-curupeté" são capturados nos varjões. Por outro lado, no "verão" é alta a citação da captura dos peixes na pedreira e calha principal do rio (Tabela 2).

\section{Discussão}

O Conhecimento Ecológico Tradicional (CET) dos pescadores entrevistados forneceu informações detalhadas sobre a composição da dieta dos pacus. Isso porque muito do conhecimento foi adquirido por meio de experiências resultantes das pescarias e de observações do ambiente que exploram. As observações das características do ambiente e as transformações decorrentes da variação entre os períodos ("inverno" e "verão") permitem a compreensão a respeito dos hábitats e áreas de alimentação dos pacus. Isso auxilia na seleção e definição das técnicas para capturar os peixes, o que é de suma importância para o êxito das pescarias. Por exemplo, no caso dos "pacu-cumaru", "pacu-ferro", "pacu-flaviano", "pacu-mafurá", "pacubranco" e "pacu-curupeté", a pesca no rio Araguari é realizada, principalmente, com caniço e trapinho (linha e anzol), utilizando como isca frutos/sementes, especialmente no período de inverno (SOARES et al., 2012). Outro aspecto a ser mencionado é o conhecimento sobre o período de disponibilidade dos frutos/sementes utilizados como isca, uma vez que as arbóreas citadas pelos pescadores têm frutificação sazonal e, em geral, associada com fluviosidade e pluviosidade da região (JUNK, 1980; GOULDING, 1980; CORREA et al., 2007). A importância da observação das características do ambiente e dos hábitos alimentares dos peixes para o sucesso nas pescarias também tem sido percebida pelos pescadores no complexo lago Grande de Manacapuru (REBELO et al., 2010), no rio Xingu (CARVALHO JUNIOR et al., 2011), e no rio Juruá (PORTO BRAGA; REBÊLO, 2014).

Em termos de composição da dieta, com base no relato dos pescadores, é possível sugerir que os seis pacus apresentam tendência ao regime alimentar herbívoro, alimentando-se de partes vegetais (folhas, flores, frutos, sementes), principalmente, de frutos/ sementes das arbóreas que margeiam o rio, além de perifíton e animais (invertebrados). Com base nas informações detalhadas pelos pescadores, ao se considerar o regime alimentar segundo o período do ciclo hidrológico, pode ser sugerido o regime herbívoro para o "inverno" e onívoro para o "verão". O fato de pacus consumirem basicamente vegetais, mas incluírem itens de origem animal no "verão", tais como invertebrados, é particularmente comum entre os pacus (GOULDING, 1980; BOUJARD et al., 1990).

Santos et al. (1997), estudando a dieta de "pacuflaviano" (= pacu-cana, Mylesinus paraschomburkgii) em rios da Amazônia, inclusive o Araguari, relatam esse peixe como herbívoro, ingerindo quase exclusivamente partes vegetativas da planta aquática rupestre da família Podostemacea (plantas de corredeiras). Para Boujard et al. (1990), o "pacu-ferro" (Myleus ternetzi) é estritamente vegetariano (herbívoro) e se alimenta de semente e folhas. Os mesmos autores relatam que o "pacu-cumaru" (Prosomyleus rhomboidales $=M$. rhomboidalis) também é herbívoro e se alimenta de vários tipos de sementes. $\mathrm{O}$ mesmo regime alimentar foi designado para o "pacu-mafurá" ( $M$. asterias) (CORREA; WINEMILLER, 2014) e o "pacu-curupeté" (T. trilobatus) (JÉGU; KEITH, 2005). Comparando-se as informações científicas com os relatos dos pescadores é possível concluir que há similaridades entre as informações para o período de "inverno". Por outro lado, apesar de não ter sido encontrado relato na literatura 
científica sobre a ingestão de invertebrados no período de "verão", é possível considerar a possibilidade de onivoria para esses peixes por causa da disponibilidade de invertebrados associados às plantas aquáticas.

Alterações na composição da dieta de peixes em águas tropicais podem ser regidas por modificações sazonais do hábitat, considerando que períodos distintos do ciclo hidrológico dispõem de diferentes condições abióticas e de ofertas de alimento (GOULDING, 1980). No inverno, ocorre o alagamento de extensas áreas de terra, expandindo o ambiente aquático e permitindo aos peixes acesso a uma grande quantidade de matéria orgânica, proveniente da vegetação terrestre inundada, que é utilizada como fonte de alimento pelos peixes (GOULDING, 1980; JUNK, 1980). Já no verão, a redução das áreas alagadas limita a disponibilidade de alimentos, tornando-os restritos (GOULDING, 1980). Essa dinâmica reflete na sazonalidade da dieta (GOULDING, 1980) e isso pode explicar o detalhamento da composição da dieta dos pacus descrita pelos pescadores. No rio Araguari, o "inverno" é marcado pela alta pluviosidade que promove a alagação de grandes áreas marginais, disponibilizando, dessa forma, hábitats que fornecem abrigo e alimentação para os peixes (SOARES et al., 2012). E a dieta herbívora dos pacus, baseada em frutos/sementes que caem na água da vegetação circundante, está exclusivamente relacionada ao seu acesso à floresta inundada (GOULDING, 1980; JUNK, 1980, CORREA et al., 2007).

No período do "inverno" (fevereiro a maio), é alta a disponibilidade de frutos/semente, pois corresponde ao período de frutificação das árvores que circundam as margens do rio Araguari, tais como o taperebá (novembro a março; FREITAS et al., 2010), andiroba (março a maio; FREITAS et al., 2010; 2013), sarão (dezembro a março, segundo os pescadores), ingá (frutos quase o ano todo, com picos em março, maio, agosto/setembro e outubro/janeiro; FALCÃO; CLEMENT, 2000; SILVA et al., 2007), seringa (agosto a maio; MAIA, 2001), jenipapo (março a junho, podendo produzir em outras épocas; MAIA, 2001) e imbaúba (fevereiro a agosto; MAIA, 2001; SALMAN et al., 2008; SILVA JÚNIOR et al., 2013). Portanto, os pacus estão consumindo recursos alimentares derivados da floresta inundada, no período que corresponde à alta disponibilidade. Os frutos/sementes, segundo os pescadores, têm forte contribuição na composição da dieta, sendo o alimento mais consumido durante o "inverno".

Outro fato importante a ser destacado é o relato dos pescadores sobre a andiroba ser consumida pelos pacus ainda no período de verão, ou seja, fora do seu período de frutificação. Isso pode ser explicado pela longevidade natural da semente, que varia entre 2 e 3 meses após disseminação, mas com o acondicionamento adequado a longevidade pode chegar a 7 meses (VIANNA, 1982).

Para essas etnoespécies, a capacidade de se alimentar de frutos/sementes é o resultado de adaptações morfológicas, fisiológicas e comportamentais adquiridas por alguns peixes Characiformes (CORREA et al., 2007). Os dentes, por exemplo, são, em geral, molariformes, grandes e multicuspidados, especialmente adaptados para quebrar e esmagar os frutos e sementes, sobretudo na fase adulta (RESENDE et al., 1998). Certamente, isso reflete em estratégias de sobrevivência comuns em ambientes inundáveis, conforme já foi observado nos pacus do Pantanal (RESENDE et al., 1998) e do Amazonas (GOULDING, 1980; MÉRONA; RANKINDE-MERONA, 2004).

No "verão", a retração da área alagada restringe os hábitats e força os peixes a migrarem e permanecerem na calha principal do rio (JUNK, 1980). Diante dessas modificações, é possível explicar as citações dos pescadores sobre esse período, sugerindo o regime alimentar onívoro, baseado em animais (cupins, gafanhotos), vegetais, no perifíton (denominado localmente de "limo", que está fixado nas pedras e no fundo do rio) e, em menores quantidades, em frutos/sementes. Segundo os pescadores, os peixes são capturados na calha do rio onde ocorrem extensos substratos rochosos nas cachoeiras e corredeiras. Esses ambientes são ocupados por muitas espécies de Podostemaceae (planta aquática), favorecendo o estabelecimento de uma fauna variada de invertebrados (SANTOS et al., 1997; TAVARES et al., 2006) que fornece itens para os peixes em período de escassez de alimentos.

Os relatos dos pescadores sobre a composição da dieta estão, em parte, de acordo com o descrito na literatura 
científica. No Amazonas, estudos etnoecológicos sobre regime alimentar de várias espécies de peixes no Baixo Juruá (PORTO BRAGA; REBÊLO, 2014), no lago Janauacá (BATISTELLA et al., 2005) e em Manacapuru (REBELO et al., 2010; LIMA; BATISTA, 2012) têm demonstrado que pescadores possuem um extenso conhecimento, relacionado, inclusive, com o ciclo hidrológico. Os pescadores acompanham cotidianamente as modificações do ambiente, e a percepção espaçotemporal possibilita-lhes conhecer as diferenças na composição da dieta. O CET dos pescadores gerou informações detalhadas sobre a dieta dos pacus "flaviano", "ferro", "mafurá" e "curupeté", no período de "verão", ainda não relatadas na literatura consultada.

Segundo os pescadores, seis principais locais de pesca se destacam ao longo do ciclo hidrológico. A sazonalidade traz o aumento da área alagada no "inverno", que amplia o ambiente e disponibiliza novos hábitats. Nessa época, os pacus procuram os varjões, onde as alagações marginais facilitam o acesso dos peixes a áreas consideradas pelos pescadores como de abrigo, alimentação e reprodução, sendo o local mais utilizado pelos pescadores para a pesca dos pacus. No "verão", a redução do nível d'água obriga os peixes a se movimentarem em busca de locais apropriados, mais profundos, como a calha do rio. Além disso, é nesse período que as pedreiras e cachoeiras ficam visíveis, locais escolhidos pelos pescadores para a prática da atividade com caniço, em cima das pedras, próximo à queda d'água. Esse tipo de pesca está detalhado em SOARES et al. (2012). Portanto, a diferenciação na utilização dos locais de pesca reflete a importância da percepção do ambiente e de suas variações sazonais na produção pesqueira (MALDONADO, 2000). A diferença sazonal de locais de pesca também tem sido relatada em estudos com pescadores sobre o acará-disco na RDSPiagaçu-Purus (ROSSONI et al., 2014), acará-açu no Praacuúba (DAADDY, 2012), vários peixes no lago Grande de Manacapuru (REBELO et al., 2010), no rio Madeira (DORIA et al., 2012) e sobre a pesca de bagres em Manacapuru e Iranduba (BRAGA, 2001).

Para as etnoespécies de pacu, a estreita relação de alimentação com os recursos provenientes da floresta circundante e matas ciliares reforça a importância no que diz respeito à conservação da biodiversidade, tanto da vegetação como dos animais, como, por exemplo, peixes que compartilham o mesmo ambiente. Nesse contexto, o CET pode ser um grande aliado para proteção de hábitats e de espécies econômica e culturalmente importantes (OLIVEIRA; SILVA, 2013). No caso dos pacus, o grau de refinamento do entendimento dos pescadores da FLONA-AP sobre as relações ecológicas dos ambientes e, especificamente, sobre o varjão, como local de alimentação e proteção, merece um olhar diferenciado quanto à sua conservação. Portanto, a inclusão do CET dos pescadores é um caminho a ser escolhido na tomada de decisões sobre manejo e conservação do recurso pesqueiro na FLONA-AP.

\section{Agradecimentos}

Agradecemos à Conservação Internacional do Brasil (CI), à Walmart Brasil e ao Instituto Chico Mendes de Conservação da Biodiversidade (ICMBio), pelo apoio no âmbito do projeto Biologia e etnoecologia da ictiofauna na Floresta Nacional do Amapá. Aos pescadores, pela disponibilidade em participar do referido estudo. Aos especialistas Michel Jegu - Institut de Recherche pour le Développement (IRD-France) e MSc. Marcelo Costa Andrade (Doutorando/UFPA), que realizaram a identificação dos pacus. Aos professores Luciano Araújo Pereira, Driss Wagner Pantoja Pena, Breno Marques da Silva e Silva da Universidade do Estado do Amapá e Kleber Pires, que identificaram os frutos. Finalmente, agradecimento aos avaliadores, pelas sugestões que melhoraram a qualidade do manuscrito.

\section{Referências}

ALBUQUERQUE, U. P. de.; LUCENA, R. F. P. Métodos e técnicas para coleta de dados. In: ALBUQUERQUE, U. P. de.; LUCENA, R. F. P. de. (Org.). Método e técnicas na pesquisa Etnobotânica. Recife: Livro Rápido/NUPEEA, 2004. p. 37-62.

BARAÚNA, T. Gestão de Unidade de Conservação e Território: uma experiência em Rondônia. Revista de Educação, Cultura e Ambiente, Porto Velho, v. 4, n. 21, p. 20-29, 2000.

BARBOZA, R. S. L.; PEZZUTI, J. C. B. Etnoictiologia dos pescadores artesanais da Resex Marinha Caeté-Taperaçu, Pará: aspectos relacionados com etologia, usos de hábitat e migração de peixes da família Sciaenidae. Sitientibus, Série Ciências Biológicas, Feira de Santana, v. 11, n. 2, p. 133-141, 2011. 
BARROS, F. B. Etnoecologia da pesca na Reserva Extrativista Riozinho do Anfrízio, Terra do Meio, Amazônia Brasil. Amazônica Revista de Antropologia, Belém, v. 4, n. 2, p. 286-312, 2012.

BARROS, J. F.; RIBEIRO, M. O. A. Aspectos sociais e conhecimento ecológico tradicional na pesca. In: BARTHEM, R. B.; FABRÉ, N. (Ed.). O manejo da pesca dos grandes bagres migradores: piramutaba e dourado no eixo Solimões-Amazonas. Manaus: Pro Várzea, 2005. p. 31-46.

BAILEY, K. D. Methods of social research. New York: Free Press, 1987. 533 p.

BATISTELlA, A. M.; CASTRO, C. P.; VALE, J. D. Conhecimento dos moradores da comunidade de Boas Novas, no Lago Janauacá Amazonas, sobre os hábitos alimentares dos peixes da região. Acta Amazonica, Manaus, v. 1, n. 35, p. 51-54, 2005.

BEGOSSI, A. Caiçaras, caboclos and natural resources: rules and scale patterns. Ambiente \& Sociedade, Campinas, n. 5, p. 55-67, 1999.

BEGOSSI, A. (Org.). Ecologia de pescadores da Mata Atlântica e da Amazônia. São Paulo: Hucitec: Nepam/Unicamp: Nupaub/ USP: FAPESP, 2004. 332 p.

BERKES, F. Sacred ecology: traditional ecological knowledge and resource management. Philadelphia: Taylor and Francis, 1999. 209 p.

BERKES, F.; COLDING, J.; FOLKE, C. Rediscovery of traditional ecological knowledge as adaptive management. Ecological Applications, Ithaca, v. 10, n. 5, p. 1251-1262, 2000.

BEZERRA, P. E.; CUNHA, B. C. C.; DEL'ARCO, J. O.; DRAGO, V. A.; MONTALVAO, R. M. G.; EULALIO, H.; PRADO, P.; AMARAL FILHO, Z. P.; NOVAES, A. S.; VIEIRA, P. C.; FRAGA, A. Y. C.; COSTA, J. R. S.; SALGADO, L. M. G.; BRAZAO, J. E. M. Projeto de zoneamento de recursos naturais da Amazônia Legal. Rio de Janeiro: IBGE/Departamento de Recursos Naturais e Estudos Ambientais, 1990. 212 p.

BOUJARD, T.; SABATIER, D.; ROJAS-BELTRAN, R.; PREVOST, M.-F.; RENNO, J.-F. The food habits of three allochthonous feeding characoids in French Guiana. Revue Ecologie (Terre et Vie), Paris, v. 45, p. 247-258, 1990.

BRAGA, T. M. P. Pressão de exploração sobre grandes bagres (Siluriformes) na Amazônia Central: municípios de Iranduba e Manacapuru, Amazonas. 2001. 105 f. Dissertação de Mestrado (Mestrado em Biologia de Água Doce e Pesca Interior) - Instituto Nacional de Pesquisas da Amazônia/ Fundação Universidade do Amazonas, Manaus. 2001.

BRANDÃO, F. C.; SILVA, L. M. A. Conhecimento ecológico tradicional dos pescadores da Floresta Nacional do Amapá. Uakari, Belém, v. 4, n. 2, p. 55-66, 2008.

CARVALHO-JÚNIOR, J. R.; FONSECA, M. J. C.; SANTANA, A. R.; NAKAYAMA, L. O conhecimento etnoecológico dos pescadores yudjá, Terra Indígena Paquiçamba, Volta Grande do Rio Xingu, PA. Tellus, Campo Grande, v. 11, n. 21, p. 123-147, 2011.

CORREA, S. B.; WINEMILLER, K. O.; LÓPEZ-FERNÁNDEZ, H.; GALETTI, M. Evolutionary perspectives on seed consumption and dispersal by fishes. BioScience, Berkeley, v. 57, n. 9, p. 748$756,2007$.

CORREA, S. B.; WINEMILLER, K. O. Niche partitioning among frugivorous fishes in response to fluctuating resources in the Amazonian floodplain forest. Ecology, Nova York, v. 95, n. 1, p. 210-224, 2014.
COSTA-NETO, E. M.; MARQUES, J. G. W. Etnoictiologia dos pescadores artesanais de Siribinha, município de Conde (Bahia): aspectos relacionados com a etologia dos peixes. Acta Scientiarum, Maringá, v. 22, n. 2, p. 553-560, 2000.

CUNHA, F. C.; SOARES, M. G. M.; FRAXE, T. J. P. O Etnoconhecimento dos pescadores sobre a reprodução do tucunaré (Cichla spp.) no Lago Grande de Manacapuru, AM. Saber do Norte, Manaus, v. 2, n. 2, p. 27-32, 2010.

DAADDY, M. D. V. Caracterização da pesca e etnobiologia do apaiari Astronotus ocellatus (Agassiz, 1831), no município de Pracuúba estado do Amapá, como subsídio à piscicultura. 2012. 107 f. Dissertação (Mestrado em Biodiversidade Tropical) Universidade Federal do Amapá, Macapá. 2012.

DIEGUES, A. C. (Org.). Etnoconservação: novos rumos para a proteção da natureza nos trópicos. São Paulo: HUCITEC: NUPAUB, 2000. 290 p.

DORIA, C. R. C.; RUFFINO, M. L.; HIJAZI, N. C.; CRUZ, R. L. A pesca comercial na bacia do rio Madeira no estado de Rondônia, Amazônia brasileira. Acta Amazonica, Manaus, v. 42, n. 1, p. 29 40, 2012.

FALCÃO, M. de A.; CLEMENT, C. R. Fenologia e produtividade do ingá-cipó (Inga edulis) na Amazônia Central. Acta Amazonica, Manaus, v. 30, n. 2, p. 173-180, 2000.

FRASER, D. J.; COON, T.; PRINCE, M. R.; DION, R.; BERNATCHEZ, L. Integrating traditional and evolutionary knowledge in biodiversity conservation: a population level case study. Ecology and Society, Nova Scotia, v. 11, n. 2, 2006. Disponível em: <http://www.ecologyandsociety.org/vol11/iss2/ art4/>. Acesso em: 15 fev. 2015.

FREITAS, J. L.; SANTOS, A. C.; SILVA, R. B. L.; RABELO, F. G.; SANTOS, E. S.; SILVA, T. L. Fenologia reprodutiva da espécie Carapa guianensis Aubl. (Andirobeira) em ecossistemas de terra firme e várzea, Amapá, Brasil. Biota Amazônia, Macapá, v. 3, n. 1, p. 31-38, 2013.

FREITAS, J. L.; SANTOS, M. M. L. S.; OLIVEIRA, F. de A. Fenologia reprodutiva de espécies potenciais para arranjo em sistemas agroflorestais, na Ilha de Santana, Amapá. Amazonian Journal of Agricultural and Environmental Sciences, Belém, v. 53, n. 1, p. 78-86, 2010.

GOULDING, M. The fishes and the forest: explorations in Amazonian natural history. Califórnia: University of California Press, 1980. 280 p.

IBGE - INSTITUTO BRASILEIRO DE GEOGRAFIA E ESTATÍSTICA. Censo demográfico 2010. 2010. Disponível em: $<$ http://www.ibge.gov.br/cidadesat/topwindow.htm?1>. Acesso em: 25 nov. 2012.

ICMBIO - INSTITUTO CHICO MENDES DE CONSERVAÇÃO DA BIODIVERSIDADE. Plano de Manejo da Floresta Nacional do Amapá. Vol. I. Macapá: ICMBIO, 2011. 220 p.

JÉGU, M.; KEITH, P. Threatened fishes of the world: Tometes lebaili (Jégu, Keith \& Belmont-Jégu 2002) (Characidae: Serrasalminae). Environmental Biology of Fishes, New York, v. 72, n. 4, p. 378, 2005.

JOHANNES R. E. Integrating traditional ecological knowledge and management with environmental impact assessment. In: INGLIS, J. T. (Ed.), Traditional ecological knowledge: concepts and cases. Ottawa: International Development Research Centre. 1993, p. 33-40. 
JOHANNES, R. E. The case for data-less marine resource management: examples from tropical nearshore fin fisheries. Trends in Ecology and Evolution, Cambridge, v. 13, p. 243-246, 1998

JUNK, W. J. Áreas inundáveis: um desafio para a limnologia. Acta Amazonica, Manaus, v. 10, n. 4, p. 775-795, 1980.

LIMA, L. G.; BATISTA, V. S. In search of traditional bio-ecological knowledge useful for fisheries co-management: the case of jaraquis Semaprochilodus spp. (Characiformes, Prochilodontidae) in Central Amazon, Brazil. Journal of Ethnobiology and Ethnomedicine, London, v. 6, p. 9, 2010

LIMA, L. G.; BATISTA, V. S. Estudos etnoictiológicos sobre o pirarucu Arapaima gigas na Amazônia Central. Acta Amazonica, Manaus, v. 42, n. 3, p. 337-344, 2012.

MAIA, L. M. A. Frutos da Amazônia: fonte de alimento para peixes. Manaus: SEBRAE-AM, 2001.143 p.

MALDONADO, S. C. A caminho das pedras: percepção e utilização do espaço na pesca simples. In: DIEGUES, A. C. (Org.). A imagem das águas. São Paulo: HUCITEC, 2000. p. 59-68.

MARQUES, J. G. W. Pescando pescadores: etnoecologia abrangente no Baixo São Francisco alagoano. São Paulo: NUPAUB, Universidade de São Paulo, 1995. 285 p.

MÉRONA, B.; RANKIN-DE-MERONA, J. Food resource partitioning in a fish community of the central Amazon floodplain. Neotropical Ichthyology, Porto Alegre, v. 2, n. 2, p. 75-84, 2004.

OLIVEIRA, G. Z.; SILVA, C. J. Conhecimento ecológico tradicional de pescadores profissionais sobre peixes da baía Caiçara, Pantanal de Mato Grosso, Cáceres, Brasil. Revista Brasileira de Zoociências, Juiz de Fora, v. 15, n. 1,2,3, p. 171-181, 2013.

PEDROSO JUNIOR, N. N.; SATO, M. Ethnoecology and conservation in protected natural areas: incorporating local knowledge in Superagui National Park management. Brazilian Journal of Biology, São Carlos, v. 65, n. 1, 2005.

PEREIRA, L. A.; SENA, K. S.; SANTOS, M. R.; NETO, S. V. C. A. Aspectos florísticos da FLONA do Amapá e sua importância na conservação da biodiversidade. Revista Brasileira de Biociências, Porto Alegre, v. 5, n. 2, p. 693-695, 2007.

PORTO BRAGA, T. M.; REBÊLO, G. H. Conhecimento tradicional dos pescadores do baixo rio Juruá: aspectos relacionados aos hábitos alimentares dos peixes da região. Interciencia, Caracas, v. 39, n. 9, p. 659-665, 2014.

RAMIRES, M.; MOLINA, S. M. G.; HANAZAKI, N. Etnoecologia caiçara: o conhecimento dos pescadores artesanais sobre aspectos ecológicos da pesca. Biotemas, Florianópolis, v. 20, n. 1, p. 101113, 2007.

REBELO, S. R. M.; FREITAS, C. E. C.; SOARES, M. G. M. Fish diet from Manacapuru Big Lake complex (Amazon): an approach starting from the traditional knowledge. Biota Neotropica, Campinas, v. 10, n. 3, p. 39-44, 2010.

RESENDE, E. K.; PEREIRA, R. A. C.; ALMEIDA, V. L. L. de; SILVA, A. G. Peixes onívoros da planície inundável do rio Miranda, Mato Grosso do Sul, Brasil. Corumbá: EMBRAPACPAP, 1998. 44 p.
ROSSONI, F.; FERREIRA, E.; ZUANON, J. A pesca e o conhecimento ecológico local dos pescadores de acará-disco (Symphysodon aequifasciatus, Pellegrin 1904: Cichlidae) na Reserva de Desenvolvimento Sustentável Piagaçu-Purus, baixo rio Purus, Brasil. Boletim do Museu Paraense Emílio Goeldi, Belém, v. 9, n. 1, p. 109-128, 2014.

ROTTA, E.; CARVAlho, L. C; ZONTA, B. M. Manual de prática de coleta e herborização de material botânico. Colombo: Embrapa Florestas, 2008. 20 p.

RUDDLE, K. The transmission of traditional ecological knowledge. In: INGLIS, J. T. (Ed.). Traditional ecological knowledge: concepts and cases. Ottawa: International Development Research Centre, 1993. p. 17-31.

SALMAN, A. K. D.; LÓPEZ, G. F. Z.; BENTES-GAMA, M. M.; ANDRADE, C. M. S. Espécies arbóreas nativas da Amazônia Ocidental Brasileira com potencial para arborização de pastagens. Porto Velho: Embrapa Rondônia, 2008. 20 p.

SANTOS, G. M.; PINTO, S. S.; JEGU, M. Alimentação do pacucana Mylesinus paraschomburgkiì (Teleostei, Serrasalmidae) em rios da Amazônia brasileira. Revista Brasileira de Biologia, São Carlos, v. 57, n. 2, p. 311-315, 1997.

SANTOS, J. Conhecimento etnoictiológico e a sua contribuição referente ao defeso de espécies de peixe de interesse comercial no município de Pracuuba, Amapá, Brasil. 2012. 69 f. Monografia (Graduação em Engenharia de Pesca) - Universidade do Estado do Amapá, Macapá. 2012.

SILVA, F. R.; MONTAG, L. F. Etnoecologia de peixes em comunidades ribeirinhas da Floresta Nacional de Caxiuanã, município de Melgaço-PA. In: SEMINÁRIO ESTAÇÃO CIENTIFICA FERREIRA PENA: dez anos de pesquisa na Amazônia - contribuição e novos desafios. 6, 2003, Belém. Anais... Belém: MPEG. 2003. Versão eletrônica.

SILVA, R. A.; NASCIMENTO, D. B.; DEUS, E. G.; SOUZA, G. D.; OLIVEIRA, L. P. S. Hospedeiros e parasitóides de Anastrepha spp. (Diptera: Tephritidae) em Itaubal do Piririm, estado do Amapá. Ciência Rural, Santa Maria, v. 37, n. 2, p. 557-560, 2007.

SILVA JÚNIOR, C. P. A. S.; GOMES, F. A.; FARINATTI, L. H. E.; LAMBERTUCCI, D. M.; MOREIRA, J. G. do V. Qualidade do feno da rama da mandioca (Manihot sculenta Crantz) e folha de embaúba (Cecropia pachystachya) armazenados por quatro meses na Amazônia Ocidental, Acre, Brasil. Enciclopédia Biosfera, Goiânia, v. 9, n. 17, p. 2221-2230, 2013.

SILVANO, R. A. M.; SILVA, A. L.; CERONI, M.; BEGOSSI, A. Contributions of ethnobiology to the conservation of tropical rivers and streams. Aquatic Conservation: Marine and Freshwater Ecosystems, Malden, v. 18, n. 3, p. 241-260, 2008.

SOARES, M. G. M.; CUNHA, F. C.; PRESTES, L. Biologia e etnoecologia da ictiofauna na Floresta Nacional do Amapá. Macapá: CI/ICMBio/Walmart, 2012. 99 p.

TAVARES, A. S.; ODNETZ, O.; ENRICONE, A. A família Podostemaceae em rios amazônicos e comunidades de insetos associados. Insula, Florianópolis, v. 35, p. 19-50, 2006.

VIANNA, N. G. Conservação de sementes de andiroba (Garapa guianensis). Belém: Embrapa/CPATU, 1982. 10 p. 